\title{
"Enseigner / apprendre" la prononciation autrement : une approche psychosociale musique- parole
}

Grégory Miras

\section{OpenEdition}

1 Journals

Édition électronique

URL : http://journals.openedition.org/rdlc/1508

DOI : $10.4000 /$ rdlc. 1508

ISSN : 1958-5772

Éditeur

ACEDLE

\section{Référence électronique}

Grégory Miras, «"Enseigner / apprendre" la prononciation autrement : une approche psychosociale musique-parole », Recherches en didactique des langues et des cultures [En ligne], 10-1 | 2013, mis en ligne le 07 janvier 2013, consulté le 01 mai 2019. URL : http://journals.openedition.org/rdlc/1508 ; DOI : 10.4000/rdlc. 1508

Ce document a été généré automatiquement le 1 mai 2019.

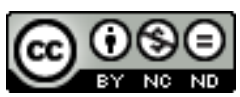

Recherches en didactique des langues et des cultures is licensed under a Creative Commons AttributionNonCommercial-NoDerivatives 4.0 International License 


\title{
"Enseigner / apprendre" la prononciation autrement : une approche psychosociale musique- parole
}

\author{
Grégory Miras
}

\section{Introduction}

1 L'enseignement / apprentissage de la prononciation est depuis longtemps un domaine de la didactique des langues délicat car "il s'agit d'une matière très particulière" (Wioland, dans Lauret, 2011 : 5). Cette particularité repose sur le fait que la prononciation fait intervenir un grand nombre de variables allant du traitement acoustique aux pressions psychosociales telles que la motivation, l'égo et les émotions. Les approches traditionnelles en didactique des langues ont tantôt favorisé l'imitation ("méthodologie directe"), les automatismes par la répétition ("le béhaviorisme") et plus récemment les interactions ("approches communicatives"). D'autres tendances, telle que la méthode verbo-tonale, ont centré l'apprentissage au "plan psychologique, corporel, psychosomatique et audio-phonatoire" (Guimbretière, $1994: 48$ ).

2 Notre article vise à mettre en avant les aspects acoustiques et psychosociaux qui interviennent dans la performance de "prononcer" une langue étrangère (LE). Nous poursuivrons notre étude par les rapports entre musique et parole sur le plan neuropsychologique afin de mesurer l'influence du type de langage sur les rapports sonindividu. Nous définirons le langage comme la faculté que les humains ont développée pour communiquer mais également pour organiser leur monde. Nous parlerons de langue comme l'objectivation descriptive du système langagier par une société et de parole (ici préférée au terme discours, plus chargé sociologiquement) comme la réalisation physique de ce langage (Saussure, 1967: 23-35). Cette réflexion s'appuiera sur une enquête quantitative par questionnaire, qui a pu montrer que les influences sociales sur les 
représentations des langages musicaux et verbaux sont importantes et prépondérantes sur des variables internes, telles que le type de pratique ou d'apprentissage. Nous terminerons, en guise de conclusion, en proposant des pistes didactiques sur un possible appui musical dans la médiation de la perception / production des sons en langue étrangère.

\section{La prononciation : de l'acoustique au psychosocial}

Le terme de prononciation en français reste définitivement ambigu, car comme le définit le Larousse en ligne (2009) c'est: "l'action, fait de prononcer un son, un signe, une lettre". La prononciation serait donc un acte physique de production sonore dans une perspective purement articulatoire, même si le Larousse ajoute une dimension individuelle : "manière propre à quelqu'un d'articuler, de rendre les sons du langage", du site Larousse en ligne (2009). Il est, par ailleurs, intéressant de constater qu'à l'origine, prononciation vient du latin pronuntiatio signifiant "publication, déclaration, annonce" (Gaffiot, 1934: 1255). Il faudra attendre le $\mathrm{XV}^{\text {lème }}$ siècle pour que le terme prononciation signifie "manière dont un mot est prononcé" mais aussi "action de lire un arrêt, un jugement" du site Centre National de Ressources Textuelles et Lexicales (2012). Le terme révélait plus un objet oral qu'un mécanisme. L'intervention du structuralisme a certainement mis en avant le caractère segmental de la prononciation plutôt que l'énoncé dans son intégralité. C'est à cette période (Cercle de Prague : 1929-1939) qu'apparaissent les deux domaines de référence que sont la phonétique et la phonologie définies respectivement comme suit: "l'étude phonétique nous dit comment sont faits les sons d'une langue, et quelles sont leurs caractéristiques acoustiques" et "une étude phonologique nous explique comment ces sons sont utilisés pour donner du sens" (Troubetzkoy, 1939: 10). Le phonème, unité minimale linguistique de l'oral (Martinet, $1970: 16$ ), peut donc accepter une double réalité : physique, telle qu'elle a été définie initialement par D. Jones (1931), mais également psychologique. Cette dernière a été mise en évidence par Baudouin de Courtenay, puis citée par Twaddell (1935: 56), Hyman (1975 : 72) et reprise par Randall (2007 : 39) :

C'est un concept abstrait, une construction mentale, un symbole qui existe dans l'esprit des locuteurs. Le défi de la psychologie et de la linguistique est d'apporter des explications sur le transfert de données brutes physiques vers des représentations abstraites symboliques de la parole.

Cette dernière vision est renforcée lorsque Krashen (1988: 35), d'obédience chomskyenne, affirme que "la prononciation est beaucoup plus ancrée dans la personnalité de l'apprenant que n'importe quel autre aspect de la langue". Par ailleurs, la réalité même du phonème, peut être mise en doute en tant que son du discours représenté dans l'esprit et que "l'on s'efforce d'atteindre" (Goody, 1994 : 276), et ce, au profit du phone, "le son concret employé dans toute émission du discours" (Ibidem : 277). Nous pouvons le dissocier en deux niveaux:physiophonique, "représentant les sons qui sont émis [réellement]" et psychophonique, "ne représentant que des phonèmes" (Ibidem). Le travail de médiation de la perception / production des sons ${ }^{1}$ aura donc pour but de tendre vers une limitation des variations entre les univers physiophonique et psychophonique, c'est-à-dire entre ce que nous entendons à travers notre filtre physiologique et cognitif et ce que nous produisons réellement. Cette distorsion n'est pas un phénomène nouveau puisque Troubetzkoy, d'obédience pragoise, nous propose dès 1939 : 
Le système phonologique d'une langue est semblable à un crible (phonological sieve) à travers lequel passe tout ce qui est dit. Seules restent dans le crible les marques phoniques pertinentes pour individualiser les phonèmes (Troubetzkoy, 1939 : 54, dans Rolland, $2011: 34$ ).

5 Notre réflexion, socioconstructiviste et émergentiste (Bertin et Narcy-Combes, 2012), sous-tend l'observation de processus physiologiques (Ellis, 1998; O'Grady, 2010) dont les ruptures ${ }^{2}$ sont déclenchées par des interactions sociales (Vygotski, 1934). Dans ce cadre, nous préférerons le terme de nativisation phonologique, qui, en référence aux travaux de Andersen (1983), met en évidence la notion de processus, plutôt que celui de crible, qui s'inscrit dans une perspective symboliste. Les termes de nativisation / dénativisation sont préférés à ceux d'assimilation / accommodation de Piaget, en raison de l'ambiguïté avec l'assimilation phonologique ${ }^{3}$. Malgré l'obédience environnementaliste pour la nativisation et constructiviste pour l'assimilation, Andersen rappelle que la nativisation est proche de l'assimilation (Andersen, 1983 : 10-11).

6 La difficulté imposée dans la médiation de la perception/production des sons en LE, est donc que la performance de "prononciation" en LE fait intervenir deux niveaux (physique et psychologique) se déroulant en même temps :

Toutes les caractéristiques de la parole ne s'additionnent pas les unes aux autres comme à l'écrit, car ces composantes de la parole ont un caractère de non-linéarité qui est la condition sine qua non de l'existence de la parole dans le temps et dans l'espace. La parole est perçue et produite de façon globale: la signification intellectuelle (contenu de l'expression) et la signification affective (contenu de l'expressivité) sont perçues en même temps. Cette structure tridimensionnelle se forme donc en fonction de la communication, un équilibre dynamique (et non-statique puisqu'il existe une mouvance permanente) s'établit entre l'individuel et le social. (Hesling, $2002: 3)$

7 Cette notion de double composition de la parole fait écho aux travaux d'Ivan Fonagy à travers le double encodage: linguistique et paralinguistique (tableau 1). Néanmoins, Fonagy mentionne à l'époque un traitement sériel, tandis que nous nous positionnerons dans la perspective de Vaissière (2008) d'un traitement "en parallèle" de ces deux codes. 


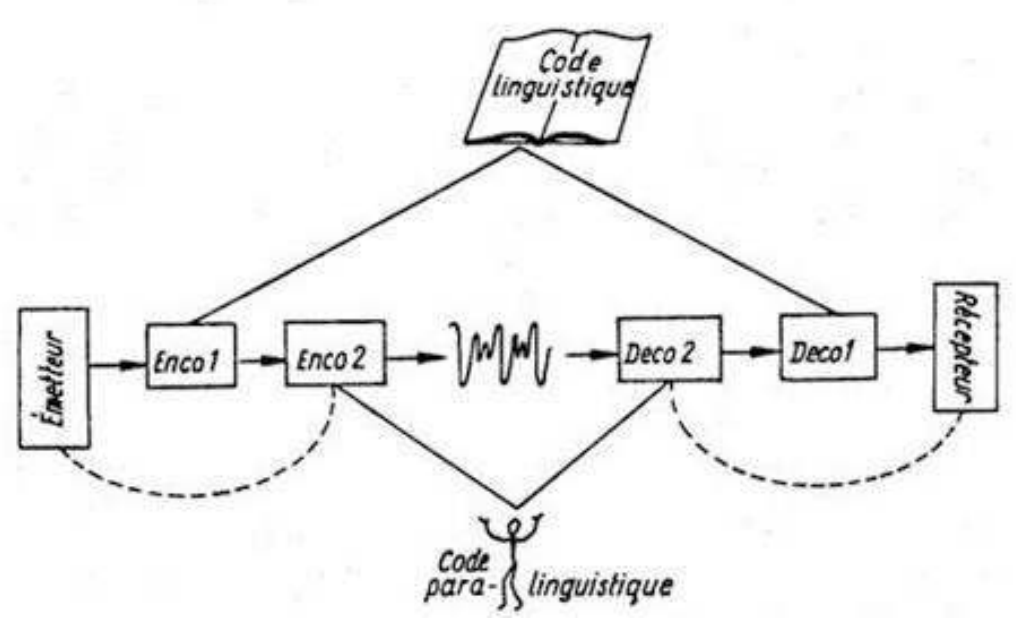

Schéma de la communication verbale à vive voix. - L'encodage linguistique est suivi d'un deuxième acte d'encodage à partir d'un « code naturel », pré-linguistique

Nous rappellerons, pour finir, que les deux réalités du son susmentionnées coexistent dans l'individu à travers la perception et la production (Randall, 2007). Ces deux phénomènes montrent une grande inter-pénétrabilité à travers la théorie motrice de la perception (Liberman et al., 1967) et dont la découverte des neurones miroirs (Rizzolatti et Sinigaglia, 2006) a permis d'apporter un support neurophysiologique.

Par conséquent, dans cette réflexion liée à la relation son-individu, il nous semble judicieux, de prendre en compte d'autres langages, comme la musique notamment. Nous verrons si ces langages possèdent des caractéristiques différentes et/ou similaires à celles de la parole, dans le but de permettre un travail ciblé en dehors du sémantisme du langage verbal.

\section{La musique instrumentale comme prisme psychosocial de la parole}

Depuis longtemps, certaines méthodes pédagogiques, plus ou moins contestées, ont mis en valeur la musique comme catalyseur d'apprentissage dans la médiation de la perception / production des sons en LE. Nous citerons pour exemple la méthode verbotonale (Renard, 2002), les "jazz chants" (Graham, 2000) ou encore la très controversée ${ }^{4}$ Méthode Tomatis (Abgrall, 2001: 145-9) reprise récemment par la Méthode Speedlingua@. De manière générale, les relations entre musique et parole se trouvent de plus en plus affaiblies dans le système éducatif moderne en raison de "l'application d'une phonétique plus instrumentale que musicale, affective et humaine" (Aubin, 2008: 101). Cependant, S. Aubin $(2008: 105)$ note 3 types de relation encore présentes:

- Recours à l'analogie entre langue et musique : "la prononciation comme la musique ne concerne que l'oreille."

- Utilisation d'expressions imagées : "des sons doux, légers, délicats, prononcer le U comme si on voulait siffler" 
- Mémorisation auditive et étude de morceaux choisis : dictées, lecture, écoute, répétitions, imitations, composition, mémorisation de morceaux choisis, reprise en chœur, etc.

11 Même si ces pratiques sont partagées par un grand nombre d'enseignants, rares sont les études didactiques ayant réussi à démontrer une efficacité significative. Nous tournerons donc cette réflexion vers l'ensemble prolifique des travaux en neurosciences de la cognition portant sur une étude comparée du traitement de la musique et de la parole, dont la plus marquante est celle de Patel (2007). Selon un travail de synthèse scientifique mené par Mithen (2007) sur les cas d'aphasie et d'amusie, il semblerait que :

les réseaux neuronaux qui opèrent le traitement langagier et musical ont un degré certain d'indépendance, il est possible de "perdre" ou ne jamais développer une des deux capacités tandis que l'autre semble normal, du moins en comparaison à la première ${ }^{5}$ (Mithen, $2007: 62$ ).

TABLEAU 2 - Une représentation modulaire de la musique (Peretz et al., 2003 : 690).

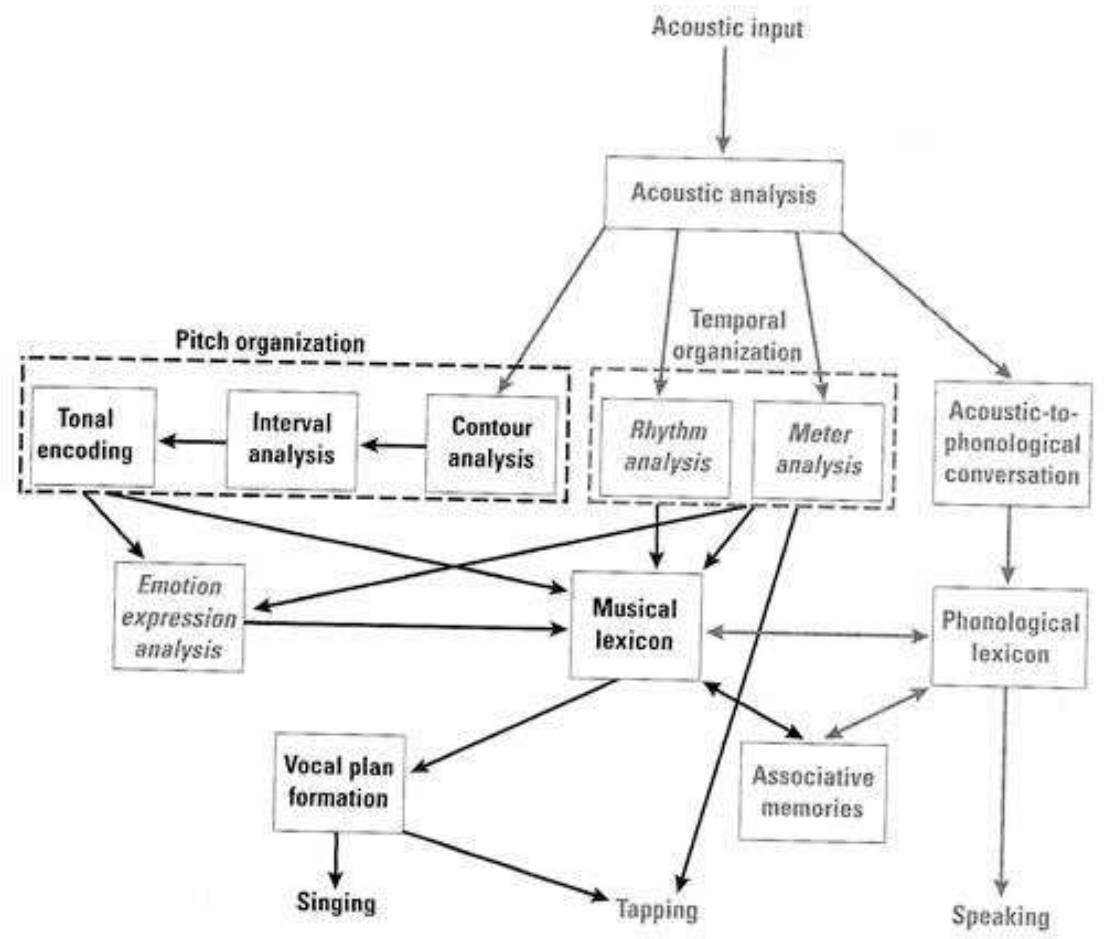

Chaque rectangle représente un bloc processuel et chaque flèche un flux d'information. Les blocs spécifiques à la musique apparaissent en noir foncé et les autres en noir / gris clair.

Néanmoins, cette "modularité" serait de nature plus complexe, dans la mesure où elle met en avant une multiplicité de traitements pour la musique et la parole. Il existerait des liens au niveau acoustique et prosodique, comme le propose Peretz et Coltheart (2003) dans le modèle ci-dessus (voir tableau 2). De plus, des travaux tendent en faveur d'une SSIRH : "shared syntactic integration resources hypothesis" (Grahn, 2011), proposant que :

la musique et le langage partagent des ressources impliquées dans les processus d'intégration structurale des événements (musicaux ou linguistiques) et des processus de mémoire de travail [...] En revanche, la musique et le langage disposeraient de réseaux neuronaux distincts pour le stockage des représentations musicales et linguistiques à long terme, 
autorisant ainsi des déficits sélectifs du traitement de la musique (i.e., amusie) et du langage (i.e., aphasie) (Hoch, $2008: 39$ ). des sons : comme de nombreuses études ont pu le démontrer (Kuhl, 2004), jusqu'à l'âge de 6-8 mois, le jeune enfant est capable de discriminer tous les contrastes phonétiques de toutes les langues, puis la perception s'organisera autour d'un "magnet effect"7 (Iverson et al., 1995). Il en va de même pour les influences de l'apprentissage précoce de la musique, dont il a été mis en évidence, par les travaux liés à la période de l'âge critique, que plus un individu commence tôt la musique, plus il est fréquent qu'il ait l'oreille absolue (Zatorre, 2003: 693). Ce phénomène implique des changements d'ordre développemental et neurophysiologique, mais aussi dans la conception cognitive de l'objet musical. De ce constat, il est communément admis que les jeunes enfants sont capables de discriminer les sons musicaux produits isolément, mais que cette faculté est perdue au profit d'un traitement par opposition pouvant se développer en "oreille relative", capacité à identifier une note en référence à une autre.

Il semblerait donc que la musique et la parole possèdent, dans l'organisation cérébrale du jeune enfant, un certain nombre de liens qui perdurent chez l'adulte. Néanmoins, l'environnement va modifier cette organisation, augmentant la distance processuelle dans le traitement de ces deux objets langagiers. En outre, l'impact socioconstructiviste implique une emphase sur le sens pour le discours tandis que la musique impliquerait plus de réponses émotionnelles (Patel, 2008 : 315-9). Ces réponses peuvent porter sur l'entre-aide (Fried et Berkowitz, 1979), la rééducation (Dalla-Bella, 2012) et la douleur (Guétin et Touchon, 2012), par exemple. Nous reprendrons la dichotomie de Damasio (2010 : 136-7) caractérisant les émotions comme "des programmes complexes et en grande partie automatisés d'action qui ont été concoctés par l'évolution" et les sentiments émotionnels comme "des perceptions composites de ce qui se passe dans notre corps et notre esprit quand nous éprouvons des émotions". Damasio définit la notion d'automatisme, en lien avec la conscience, comme "un sentiment automatique, spontané et immédiat de soi, en tant que protagoniste de l'expérience de soi-même" (2010: 198). Concernant l'apprentissage, de récentes études ont pu attester du fait que :

l'apprentissage de la musique influence le traitement des unités de base du langage, les syllabes [allant] à l'encontre d'une conception modulaire et informationnellement encapsulée du langage qui serait indépendante des autres capacités perceptives et cognitives. Ces résultats indiquent que l'apprentissage musical ne facilite pas seulement le traitement des paramètres acoustiques tel que la durée ou la fréquence, mais améliore également la perception de paramètres linguistique plus abstraits tel que le VOT $^{8}$ (Chobert et Besson, $2012:$ 71).

Neanmoins, ces travaux portent sur des enfants dont les caractéristiques cérébrales sont en pleine maturation, même s'il a été démontré que la plasticité cérébrale est toujours active à l'âge adulte (Schiffmann, 2001).

D'après de récentes études en neurosciences de la cognition, la modularité cérébrale (si elle existe) ne serait pas innée mais la résultante d'interactions entre les conditions biologiques et l'environnement social visant un traitement efficace de l'information (Berthoz, 2009). D'autre part, cette modularité ne serait pas absolue mais favoriserait des interconnexions entre les sous-domaines processuels. Nous pouvons, de ce fait, valider

Recherches en didactique des langues et des cultures, 10-1 | 2013 
certaines applications de la musique dans le cadre de l'apprentissage des langues sur le plan neurophysiologique. Il reste, cependant, à déterminer si la construction de représentations catégorisées (musique et parole), chez l'adulte, peut être un frein ou un moteur dans les applications susmentionnées. Nous avons dans ce but mis en place une enquête quantitative mesurant les représentations de musiciens et de locuteursplurilingues respectivement sur les langages musical et verbal.

\section{Enquête quantitative par questionnaire}

L'enquête quantitative présentée vise à mesurer l'influence du type de langage (musique ou parole) dans les représentations sur les relations entre l'individu et le son et ce, en comparaison à des variables telles que les modalités de pratique ou d'apprentissage. Nous tenterons, en outre, de voir si la relation au son chez les musiciens peut apporter une valeur acquisitionnelle dans la médiation de la perception / production des sons chez des locuteurs-plurilingues et ce, sur les plans intra- et inter-psychiques (émotions, interactions, cognition, etc.) tels que définis par Vygotski (1934). Nous tenons à préciser qu'une telle enquête ne reflète pas une conduite sociale réelle mais, dans notre perspective psychosociologique, met en évidence des représentations fortes pouvant se traduire en actes, en réactions ou influencer les sentiments des individus.

Il a été précisé que chaque répondant devait choisir un seul questionnaire parmi les quatre proposés, en fonction de celui qui correspondait le plus à son parcours. De plus, les musiciens ont été interrogés sur leur rapport au langage musical et les locuteursplurilingues au langage verbal uniquement. Nous avons ainsi limité le biais du fait que certains musiciens puissent être plurilingues et certains locuteurs-plurilingues, des musiciens.

\section{Matériel et méthode}

19 L'enquête a été créée sur la base d'un formulaire Googledocument $\odot$. Elle comporte 2 questionnaires différents repartis en 4 panels :

Tableau 3 - Caractéristiques des 4 questionnaires de l'enquête quantitative.

\begin{tabular}{|c|c|c|c|c|}
\hline & \multicolumn{2}{|c|}{ Panel } & Spécificité(s) & Sigle \\
\hline & 1 & Musicien & Sans cours formels de solfège & MSS \\
\hline Questionnaire & & & Avec cours formels de solfège & MAS \\
\hline & 2 & Locuteur-plurilingue & Pratique régulière LE en contexte naturel & LPR \\
\hline & & & Pratique non régulière LE en contexte naturel & LPNR \\
\hline
\end{tabular}

Les sigles signifient respectivement musiciens sans cours de solfège (MSS), musiciens avec cours de solfège (MAS), locuteurs-plurilingues avec pratique régulière en contexte naturel (LPR) et locuteursplurilingues sans pratique régulière en contexte naturel (LPNR) 
Nous définissons :

- la pratique du solfège comme une activité cristallisée autour d'une culture occidentale et de pratiques définies : rythme, déchiffrage, etc. favorisant une approche métacognitive de la musique (Tripier-Mondancin, 2008),

- un locuteur-plurilingue comme un individu pratiquant au moins deux langues à des niveaux différents (Truchot, 1994),

- la pratique régulière d'une LE en contexte naturel comme parler avec des locuteurs natifs (réel ou virtuel), voyage / séjour dans un des pays, lire des livres, regarder la télévision (Tyne, 2012).

21 Chaque questionnaire se compose de 4 parties : les informations générales, spécifiques à chaque groupe, un questionnaire à échelle d'attitude du type échelle de Likert et cinq questions à choix multiples acceptant une ou plusieurs réponses.

Tableau 4 - Exemple de correspondances entre phrases affirmatives dans le questionnaire à échelle d'attitude des 4 panels

\begin{tabular}{|l|l|l|}
\hline Panel & Musiciens (MAS/MSS) & Locuteurs plurilingues (LPR/LPNR) \\
\hline Affirmation & La timidité influence la qualité du jeu & La timidité influence la qualité de l'oral \\
\hline
\end{tabular}

Les données ont été récupérées à travers l'envoi d'un courriel diffusé à de nombreux organismes et établissements universitaires ou de promotion de la musique. L'analyse des données a été faite par l'auteur de manière manuelle ou automatique par tableur.

\section{Profil socioculturel}

L'ensemble de l'enquête regroupe 1173 répondants répartis dans les proportions du diagramme ci-dessous : 


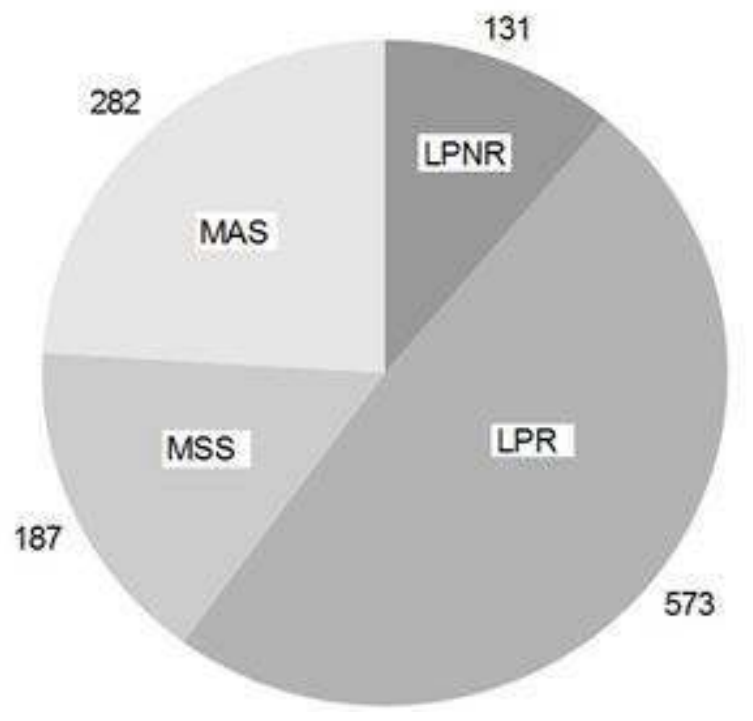

Le panel est composé majoritairement de femmes (61\%), de personnes âgées de 15 à 25 ans $(62 \%)$ ayant une nationalité occidentale et / ou vivant dans un pays occidental et / ou ayant fait leurs études dans un pays occidental (plus de $90 \%$ ) et ayant fait des études supérieures (77\%). Nous pouvons donc penser que le grand nombre de réponses permet une représentativité suffisante pour nos conclusions. Cependant, les résultats proposés devront être pris dans le cadre d'un profil socioculturel spécifique d'individus plutôt jeunes adultes, avec des représentations occidentales et un haut niveau métacognitif. Le biais du genre n'est pas retenu ici car, selon certaines études, les caractéristiques individuelles seraient plus importantes que celle du genre, à ce niveau de traitement (Daumas, 2005).

\section{Discussions}

Une première analyse des données a permis de mettre en évidence une homogénéité certaine à l'intérieur de chaque panel, les musiciens (MAS et MSS) d'un côté et les locuteurs-plurilingues (LPR et LPNR) de l'autre, et ce malgré les variables étudiées : l'apprentissage du solfège et la pratique d'au moins une langue étrangère en contexte naturel. Cette partie étudiera plus particulièrement les ressemblances ou différences qui peuvent exister dans les représentations sur les formes sonores, pour les langages étudiés (la musique et le discours), afin de voir si ces relations dépassent les caractéristiques de la frontière psychosociologique entre ces domaines. De plus, nous pouvons comparer ces données étant donné l'homogénéité sur le plan socioculturel de ces panels en termes générationnel (15-25 ans), culturel (culture occidentale) et cognitif (haut niveau d'étude). L'analyse portera sur 4 dimensions : le rôle du locuteur / musicien, les aspects intra- et interpsychiques, et émotionnels. 


\section{Rôle du locuteur / musicien}

Conformément à ce qui a été mentionné ci-dessus, nous retrouvons dans cette thématique une homogénéité des réponses chez les musiciens et chez les locuteursplurilingues (tableau 6).

Tableau 6 - Résultats comparatifs en pourcentages de réponses au questionnaire à échelle d'attitude pour deux affirmations sur le rôle du locuteur / musicien.

\begin{tabular}{|l|l|c|c|c|c|c|}
\hline \multicolumn{2}{|l|}{} & $\begin{array}{c}\text { Tout à } \\
\text { fait }\end{array}$ & Plutôt & Moyen & $\begin{array}{c}\text { Plutôt } \\
\text { pas }\end{array}$ & $\begin{array}{c}\text { Pas du } \\
\text { tout }\end{array}$ \\
\hline $\begin{array}{l}\text { Parler comme un natif est le plus important pour } \\
\text { bien parler une langue étrangère }\end{array}$ & LPR & $\mathbf{2 2 \%}$ & $\mathbf{3 6 \%}$ & $\mathbf{2 6 \%}$ & $11 \%$ & $5 \%$ \\
\hline & LPNR & $\mathbf{2 0 \%}$ & $\mathbf{4 4 \%}$ & $\mathbf{2 7 \%}$ & $\mathbf{8} \%$ & $2 \%$ \\
\hline $\begin{array}{l}\text { Transmettre des émotions est le plus important } \\
\text { pour bien parler une langue étrangère }\end{array}$ & LPR & $18 \%$ & $\mathbf{5 0 \%}$ & $\mathbf{2 5 \%}$ & $6 \%$ & $2 \%$ \\
\hline $\begin{array}{l}\text { Un bon musicien apporte sa personnalité dans } \\
\text { l'œuvre qu'il interprète }\end{array}$ & MAS & $\mathbf{5 7 \%}$ & $\mathbf{3 7 \%}$ & $5 \%$ & $1 \%$ & $0 \%$ \\
\hline $\begin{array}{l}\text { Un bon musicien doit seulement reproduire les } \\
\text { intentions du compositeur }\end{array}$ & MAS & $3 \%$ & $11 \%$ & $\mathbf{3 4 \%}$ & $\mathbf{2 7 \%}$ & $\mathbf{2 5 \%}$ \\
\hline & LPNR & $15 \%$ & $\mathbf{3 7 \%}$ & $\mathbf{3 4 \%}$ & $12 \%$ & $3 \%$ \\
\hline
\end{tabular}

Au-delà de notre première analyse, nous pouvons constater que le rapport à la "norme" est totalement différent dans un groupe et dans l'autre. La partition est posée en tant que norme, étant donné qu'elle reflète les intentions de l'auteur. L'œuvre initiale n'existe que dans l'esprit du compositeur. Dans le cas du discours, le locuteur natif est devenu dans la doxa une des normes prédominantes. Il est intéressant de constater que même si les deux groupes (locuteurs-plurilingues et musiciens) affirment une tendance forte à l'intégration d'une charge émotionnelle et personnelle dans l'acte sonore: les locuteurs-plurilingues chercheraient à atteindre la norme du "natif", tandis que les musiciens devraient s'écarter de la norme écrite (la partition), se positionnant ainsi comme des interprètes, et non comme des exécutants (Corbel, 2005: 78). Le musicien ne pourrait intégrer sa 
personnalité dans l'œuvre que s'il se détache des intentions premières du compositeur afin d'y intégrer son intentionnalité émotionnelle. Le locuteur-plurilingue ne voit pas d'opposition entre d'une part, son intentionnalité émotionnelle et d'autre part, le fait d'atteindre une norme externe : le "natif".

\section{Niveau intrapsychique} style de musique qu'ils jouent, que ce soit à l'aide d'une partition ou de manière improvisée. Ce sentiment est renforcé chez les musiciens ayant fait du solfège. Il est intéressant de voir que cette tendance ne se retrouve pas chez les locuteurs-plurilingues. En outre, il n'y a pas de tendance significative sur le fait que les locuteurs pensent aux différentes caractéristiques de la "langue" quand ils parlent leur(s) langue(s) maternelle (s) ni avant de lire un texte dans une langue étrangère. Néanmoins, nous pouvons remarquer que les deux panels LPR et LPNR montrent une tendance positive, à ce genre de pratique, avant d'utiliser une langue étrangère à l'oral. Il apparaîtrait par conséquent que sur le plan cognitif, la pratique de la musique et ce, même à un niveau important (MAS), reste une pratique qui demande une réflexion métacognitive liée à l'ensemble des paramètres qu'il faut maîtriser dans l'acte de jouer. La mise en son (parole) de la L1 / LM est un acte automatique, qui plus est, chez les locuteurs-plurilingues n'ayant pas de pratique régulière d'une langue étrangère. Seule la pratique orale d'une langue étrangère semble imposer chez les LPR et LPNR une réflexion métacognitive. Nous pensons que si l'affirmation avait porté sur un registre oral plus académique, se rapprochant de la pratique écrite et favorisant donc une réflexion métacognitive car moins automatique, nous aurions pu constater des tendances différentes. De plus, il semblerait que les locuteurs-plurilingues soient moins sensibles que les musiciens à leurs représentations, sur le plan intrapsychique.

Cette opposition se retrouve lorsque les musiciens montrent de l'importance dans le "bien jouer" pour la qualité du son, contrairement aux locuteur-plurilingues qui cherchent à transmettre du sens. Cependant, les 4 panels (LPR / LPNR / MAS / MSS) montrent plus d'intérêt pour le fait d'être à l'aise même s'il y a des erreurs. La norme du "bien jouer" se trouverait donc chez le locuteur-plurilingue dans une interaction réussie tandis que le musicien chercherait à ce que sa perception interne et externe coïncident le plus possible sans inclure le spectateur dans cette relation. En effet, l'enquête montre qu'une minorité des musiciens pensent que le retour positif des spectateurs est un critère d'avoir "bien joué".

Ce parallèle se retrouve lorsque nos 4 panels ont l'impression, lorsqu'ils changent de "langue" / style de musique, d'être la même personne tout en agissant différemment avec les autres (interlocuteur, spectateurs, etc.). Une minorité, dans les 4 cas, pense changer de personnalité (tableau 7).

Tableau 7 - Résultats comparatifs en pourcentages de réponses au questionnaire à échelle d'attitude pour deux affirmations sur les effets psychologiques du changement de langue / style de musique.

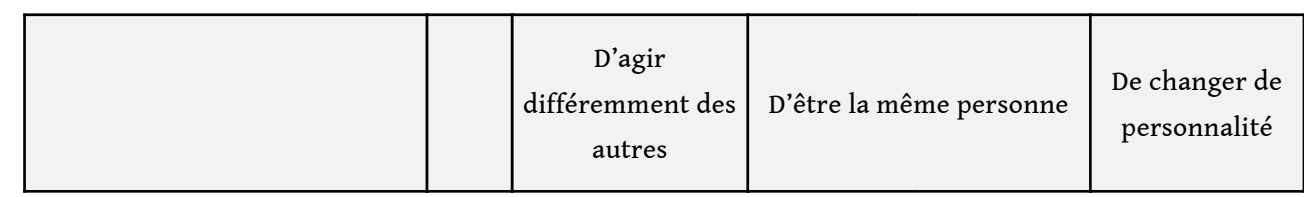

Recherches en didactique des langues et des cultures, 10-1 | 2013 


\begin{tabular}{|c|c|c|c|c|c|}
\hline $\begin{array}{l}\text { Lorsque je change de langue j'ai } \\
\text { l'impression }\end{array}$ & LPR & $37 \%$ & \multicolumn{2}{|c|}{$43 \%$} & $20 \%$ \\
\hline & LPNR & $36 \%$ & \multicolumn{2}{|c|}{$50 \%$} & $15 \%$ \\
\hline & & \multicolumn{2}{|c|}{$\begin{array}{l}\text { D'être dans le même état } \\
\text { d'esprit }\end{array}$} & \multicolumn{2}{|c|}{ De changer d'esprit } \\
\hline $\begin{array}{l}\text { Lorsque je change de langue j'ai } \\
\text { l'impression }\end{array}$ & LPR & \multicolumn{2}{|l|}{$47 \%$} & \multicolumn{2}{|c|}{$53 \%$} \\
\hline & LPNR & \multicolumn{2}{|l|}{$52 \%$} & \multicolumn{2}{|c|}{$48 \%$} \\
\hline & & $\begin{array}{c}\text { D'agir } \\
\text { différemment des } \\
\text { autres }\end{array}$ & \multicolumn{2}{|c|}{ D'être la même personne } & $\begin{array}{l}\text { De changer de } \\
\text { personnalité }\end{array}$ \\
\hline $\begin{array}{l}\text { Lorsque je change de style de } \\
\text { musique (classique, baroque, } \\
\text { moderne, folk, etc...) j'ai } \\
\text { l'impression }\end{array}$ & MAS & $45 \%$ & \multicolumn{2}{|c|}{$38 \%$} & $17 \%$ \\
\hline & MSS & $39 \%$ & & & $22 \%$ \\
\hline & & \multicolumn{2}{|c|}{$\begin{array}{l}\text { D'être dans le même état } \\
\text { d'esprit }\end{array}$} & \multicolumn{2}{|c|}{ De changer d'esprit } \\
\hline $\begin{array}{l}\text { Lorsque je change de style de } \\
\text { musique (classique, baroque, } \\
\text { moderne, folk, etc...) j'ai } \\
\text { l'impression }\end{array}$ & MAS & \multicolumn{2}{|c|}{$16 \%$} & \multicolumn{2}{|c|}{$84 \%$} \\
\hline & MSS & \multicolumn{2}{|c|}{$19 \%$} & \multicolumn{2}{|c|}{$81 \%$} \\
\hline
\end{tabular}

Les individus auraient donc une conscience stable de qui ils sont en tant que personne, quel que soit le type de production qu'ils émettent, et qu'ils souhaitent maintenir lorsqu'ils affirment vouloir garder leur identité personnelle quand ils changent de musique / "langue". Cependant, les 4 panels montrent peu d'intérêt dans la conservation de leur identité culturelle et montrent une importance significative dans l'intégration des éléments culturels de la "langue" ou des spécificités d'un style de musique. Néanmoins, nous constatons qu'un nombre très significatif ( $80 \%$ ) de musiciens (MAS et MSS) pensent changer d'état d'esprit lorsqu'ils changent de style de musique, contrairement aux locuteurs-plurilingues (LPR et LPNR) qui ne montrent pas de tendance probante (tableau 7). Il semblerait que la doxa proposant une coexistence / co-construction de la penséediscours ${ }^{9}$ favoriserait un continuum de distanciation dans la frontière entre les discours (les "langues") et par conséquent une pluralité de positionnements. À l'opposé, la pratique musicale, quel que soit le type d'apprentissage, s'organiserait en espaces 
cognitifs cohérents pour chaque type de musique que le musicien peut produire. Les musiciens s'apparenteraient à des acteurs capables de "jouer" différents rôles éphémères sans que leur identité profonde soit modifiée. Nous faisons l'hypothèse que nous pourrions retrouver ce genre de tendance chez des locuteurs lors d'un changement de registre de langue dans des contextes particuliers (situation académique versus relâchée, etc.).

Cette notion d'espace cognitif apparait également lorsque les 4 panels présentent une tendance forte concernant des sensations différentes lorsqu'ils changent de "langue" ou de style de musique. Il semblerait que cette notion se développe au-delà de la sphère purement cognitive, mais se projette également sur la sphère physique, de manière rationalisée ou non, et permettant ainsi de catégoriser un réseau de caractéristiques propres à une performance donnée (tableau 8).

Tableau 8 - Résultats comparatifs en pourcentages de réponses au questionnaire à échelle d'attitude pour deux affirmations sur les effets physiques du changement de langue / de style de musique

\begin{tabular}{|c|c|c|c|c|c|c|c|c|c|c|}
\hline & \multicolumn{5}{|c|}{ LPR } & \multicolumn{5}{|c|}{ LPNR } \\
\hline & $\begin{array}{l}\text { Tout } \\
\text { à fait }\end{array}$ & Plutôt & Moyen & $\begin{array}{c}\text { Plutôt } \\
\text { pas }\end{array}$ & $\begin{array}{c}\text { Pas } \\
\text { du } \\
\text { tout }\end{array}$ & $\begin{array}{l}\text { Tout } \\
\text { à fait }\end{array}$ & Plutôt & Moyen & $\begin{array}{c}\text { Plutôt } \\
\text { pas }\end{array}$ & $\begin{array}{l}\text { Pas } \\
\text { du } \\
\text { tout }\end{array}$ \\
\hline $\begin{array}{l}\text { Lorsque je parle/ } \\
\text { lis un texte en } \\
\text { langue étrangère, } \\
\text { je ressens des } \\
\text { différences } \\
\text { physiques } \\
\text { par rapport à } \\
\text { lorsque je parle/ } \\
\text { lis } \\
\text { un texte en } \\
\text { langue } \\
\text { maternelle }\end{array}$ & $29 \%$ & $36 \%$ & $17 \%$ & $12 \%$ & $7 \%$ & $30 \%$ & $34 \%$ & $18 \%$ & $15 \%$ & $4 \%$ \\
\hline & \multicolumn{5}{|c|}{ MAS } & \multicolumn{5}{|c|}{ MSS } \\
\hline $\begin{array}{l}\text { Lorsque je joue } \\
\text { une partition/ } \\
\text { tablure dans un } \\
\text { style, } \\
\text { je ressens des } \\
\text { différences } \\
\text { physiques } \\
\text { par rapport à } \\
\text { lorsque je joue } \\
\text { une partition } \\
\text { dans un style } \\
\text { différent }\end{array}$ & $41 \%$ & $35 \%$ & $16 \%$ & $5 \%$ & $3 \%$ & $30 \%$ & $43 \%$ & $16 \%$ & $8 \%$ & $3 \%$ \\
\hline
\end{tabular}


terminerons cette analyse intrapsychique par le constat qu'aucun des panels n'a montré de tendance significative concernant le fait de penser en même temps qu'ils parlent ou jouent de la musique. Il serait intéressant de décomposer cet aspect afin de voir, dans une future étude, si aucune tendance ne peut être mise en évidence.

\section{Niveau interpsychique et émotions}

Après avoir analysé les similitudes et différences intrapsychiques entre nos panels de musiciens et locuteurs-plurilingues, nous nous intéresserons aux plans interpsychique et émotionnel afin d'apporter un regard complémentaire. Nous pouvons tout d'abord constater que les 4 panels montrent une tendance dans une prise de conscience dans la différence entre ce qui est produit (par soi) et ce qui est perçu (par autrui). Cette prise de conscience est la plus forte chez les musiciens ayant fait du solfège, comparable chez les MSS et LPNR et enfin plus faible chez les LPR (tableau 9).

Tableau 9 - Continuum de prise de conscience entre ce qui est produit et ce qui est perçu chez les 4 panels étudiés

Prise de conscience entre ce qui est produit et ce qui est perçu

Forte

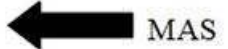

MAS

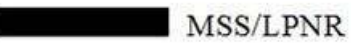

MSS/LPNR

LPR

Faible

Les MAS ont une pratique (orchestre, groupes semi-professionnel et amateur) plus variée que les MSS, ce qui peut expliquer cette plus grande sensibilité aux distorsions du son dans l'espace. Les LPNR présentent, théoriquement, une forte différence entre leurs productions en langue(s) première(s) (L1) et leurs LE, de par leur niveau, ce qui implique une plus grande prise de conscience entre ce qu'ils aimeraient produire et ce qu'ils produisent en réalité. Cette considération peut également expliquer que les LPR ont la perception la moins sensible à cette différence, puisque qu'ils peuvent valider plus facilement une interaction en LE. Par conséquent, les différences de forme sont peu pertinentes dans le caractère co-construit du sens dans l'interaction. Malgré une prise de conscience des différences entre soi et autrui, les musiciens montrent également peu d'intérêt à obtenir un retour positif des spectateurs dans le "bien jouer", tandis que les locuteurs-plurilingues veulent, une fois encore, valider l'interaction.

Tableau 10 - Résultats comparatifs en pourcentages de réponses au questionnaire à échelle d'attitude pour deux affirmations sur l'influence de la timidité sur la qualité de l'oral / jeu

\begin{tabular}{|l|c|c|c|c|c|c|c|c|c|c|}
\hline & \multicolumn{5}{|c|}{ LPR } & \multicolumn{5}{c|}{ LPNR } \\
\hline & $\begin{array}{c}\text { Tout à } \\
\text { fait }\end{array}$ & Plutôt & Moyen & $\begin{array}{c}\text { Plutôt } \\
\text { pas }\end{array}$ & $\begin{array}{c}\text { Pas du } \\
\text { tout }\end{array}$ & $\begin{array}{c}\text { Tout à } \\
\text { fait }\end{array}$ & Plutôt & Moyen & $\begin{array}{c}\text { Plutôt } \\
\text { pas }\end{array}$ & $\begin{array}{c}\text { Pas du } \\
\text { tout }\end{array}$ \\
\hline $\begin{array}{l}\text { La timidité } \\
\text { influence } \\
\text { la qualité } \\
\text { de } \\
\text { l'oral }\end{array}$ & $\mathbf{5 4 \%}$ & $30 \%$ & $8 \%$ & $5 \%$ & $3 \%$ & $\mathbf{6 4 \%}$ & $21 \%$ & $7 \%$ & $6 \%$ & $2 \%$ \\
\hline
\end{tabular}




\begin{tabular}{|l|c|c|c|c|c|c|c|c|c|c|}
\hline & \multicolumn{5}{|c|}{ MAS } & \multicolumn{3}{c|}{ MSS } \\
\hline $\begin{array}{l}\text { La timidité } \\
\text { influence la } \\
\text { qualité } \\
\text { du } \\
\text { jeu }\end{array}$ & $\mathbf{3 9 \%}$ & $37 \%$ & $12 \%$ & $7 \%$ & $4 \%$ & $\mathbf{4 2 \%}$ & $35 \%$ & $12 \%$ & $6 \%$ & $5 \%$ \\
\hline
\end{tabular}

Toutefois, les 4 panels s'accordent à reconnaitre l'influence de la timidité sur la qualité de l'oral / jeu (tableau 10).

\section{Conclusion et pistes didactiques}

À travers cette étude, nous avons pu mettre en évidence le fait que la "prononciation" fait intervenir plus qu'une réalité physique simplement (articulatoire et perceptive). L'ensemble de nos fonctions de la perception sont régies par un seul organe : le cerveau dont l'intake modifie sensiblement les informations entre l'input et l'output. Il faut donc, dans la médiation de la perception / production des sons en langue étrangère, apporter un plus grand intérêt aux aspects psychosociaux qui interviennent directement ou indirectement dans la production des sons.

Notre approche a pu mettre en évidence l'influence du type de langage (musical ou verbal) sur les représentations des individus interrogés. Il semblerait que cette influence soit prépondérante sur d'autres variables comme le type d'apprentissage et de pratique. Les musiciens cherchent à se libérer de la norme de la partition, tandis que les locuteursplurilingues tendent à vouloir s'approcher de celle du "natif". Néanmoins, dans les deux cas, il leur importe de déclencher des émotions chez autrui. Dans le cadre des représentations de sondés d'influence majoritairement occidentale de la musique, il apparaîtrait que jouer de la musique est un acte qui demande une réflexion métacognitive semblable à celle requise par les locuteurs-plurilingues dans la pratique orale d'une langue étrangère. Cependant, ces derniers semblent faire état d'un traitement plutôt automatique de la L1 qui ne se retrouve pas dans la pratique musicale, au niveau psychocognitif. Ce phénomène intervient probablement $d u$ fait que la pratique musicale occidentale moderne se fait avant tout à partir de supports écrits, limitant le sentiment d'automatisation. Dans le "bien jouer / parler", les locuteurs-plurilingues chercheraient à valider l'interaction tandis que les musiciens veulent que leurs perceptions interne et externe coïncident le plus possible. L'une des grandes différences, entre nos deux panels, réside dans le fait que les musiciens ressentent majoritairement qu'ils changent d'état d'esprit quand ils changent de musique, tandis que les locuteurs-plurilingues ne montrent pas de tendance significative concernant le changement de langue ${ }^{10}$. Toutefois, les deux semblent affirmer une stabilité, en tant qu'individu, montrant l'impact des représentations du lien discours-pensée qui conditionne cette stabilité renforcée chez les locuteurs-plurilingues.

Pour terminer, nous avons pu démontrer que les musiciens ayant fait du solfège ont une grande conscience de la différence entre ce qui est produit et ce qui est perçu, et les locuteurs-plurilingues qui ont une pratique régulière d'une LE en contexte naturel sont ceux qui en ont la conscience la plus faible. Mais cette différence serait inhibée sur le plan 
physique : les 4 panels montrent une forte conscience des différences physiques quand ils changent de langue / de style de musique. Il semblerait donc que l'objet musical favorise une prise de conscience cognitive, phénomène renforcé par l'apprentissage de la musique par le solfège et inhibée chez les locuteurs pratiquant au moins une langue étrangère en contexte naturel par leur pratique automatique. L'influence des émotions dans la mise en son est une notion partagée par tous.

Au regard de nos résultats, l'approche communicative ne semble pas être optimale pour une réflexion sur la performance de "prononciation", puisqu'elle renforce le traitement du sens plutôt qu'une prise de recul sur le son. Ce traitement apparaît comme une spécificité du discours en comparaison à d'autres langages, tels que la musique. Nous proposons donc une approche alternative, en dehors du sémantisme lexical, qui pourrait permettre un travail efficace et ciblé sur le son.

41 Les pistes didactiques, que nous proposons au terme de cette étude, s'inscrivent dans une approche par tâche développée notamment par Narcy-Combes et al. (2010) en tâches sociales (macro-tâches) et tâches d'entraînement (micro-tâches). Cependant, nous pensons que la spécificité de la médiation de la perception / production des sons en langue étrangère impose, de par son statut, un travail majoritairement en termes de micro-tâches puisque, comme nous l'avons vu, le sens prédomine sur le son dans les interactions sociales. Les tâches sociales ont pour but de faire émerger de nouvelles performances à travers des tâches réelles; or, la prononciation, même chez les natifs, reste implicite (contrairement à la syntaxe ou au lexique), ce qui limite l'émergence de nouvelles pratiques au niveau phonético-phonologique dans l'interaction.

Le travail que nous proposons sur la prononciation s'adresse à des apprenants désirant améliorer leur performance en langue étrangère en travaillant le traitement articulatoire et perceptif dans un système phonologique autre que celui de leur(s) L1. Ce travail permettra à l'individu de se sentir plus à l'aise dans sa production et de favoriser la compréhension/ interaction avec des locuteurs "natifs". Développer une approche musicale dans la médiation de la perception/ production des sons pourrait donc permettre d'augmenter, chez les locuteurs-plurilingues, une conscientisation de l'écart entre ce qu'ils pensent et ce qu'ils produisent réellement. Cette dernière pourrait reposer sur une approche métacognitive favorisant une nouvelle conception des limites penséediscours afin de prendre de la distance émotionnelle sur ce qu'impliquent des transformations de leur(s) prononciation(s). Ce travail supposerait, comme nous l'avons vu avec les musiciens, un travail recentré sur le soi avant de retourner vers autrui. Ceci pourrait passer par une prise de distance avec la / les L1, qui, comme nous l'avons montré, revêt(ent) un caractère automatique.

43 Pour finir, un travail sur la gestion des sentiments émotionnels (stress, sentiment d'insécurité, etc.) parait nécessaire. L'intentionnalité émotionnelle semble être plus consciente chez les musiciens dans leur(s) pratique(s) musicale(s) que chez les locuteursplurilingues dans leur(s) pratique(s) verbale(s). Cette conscience permettrait notamment une meilleure gestion des états émotionnels (tremblements, etc.) dans la mise en son.

44 L'ensemble de ces propositions ne sont que des pistes didactiques basées sur les représentations de nos sondés, il restera dans de futurs travaux à déterminer quels types de tâche peuvent être mises en place et dans quel ordre. 


\section{BIBLIOGRAPHIE}

Abgrall, J. M. (2001). Healing or Stealing? Medical charlatans in the New Age. New York: Algora Publishing.

Andersen, R. (1983). Pidginisation and Creolisation as Language Acquisition. Rowley : Newbury House.

Aubin, S. (2008). "Maître de langue, professeur de langue et enseignement de la musique du français". Synergies Espagne. $\mathrm{N}^{\circ} 1$.

Bertin, J.-C. \& Narcy-Combes, J.-P. (2012). "Tutoring at a distance, modeling as a tool to control chaos". In Bertin, J.-C. \& Narcy-Combes, J.-P. (Eds). Computer-Assisted Language Learning, Special issue Tutoring at a Distance. pp. 111-127.

Berthoz, A. (2009). La Simplexité. Paris : Odile Jacob.

Chobert, J. et Besson, M. (2012). "Influence de l'apprentissage de la musique sur la perception des syllabes chez les enfants normolecteurs et dyslexiques". In Bigand, E. et al. . Musique et Cerveau : nouveaux concepts, nouvelles applications. Montpellier : Sauramps Medical. pp. 61-75.

Corbel, E. (2005). "Considération sur l'interprétation chez Vladimir Jankélévitch et Igor Stravinsky". Horizons philosophiques, vol. 16, $\mathrm{n}^{\circ} 1$.

Damasio, A. (2010). L'autre moi-même : les nouvelles cartes du cerveau, de la conscience et des émotions. Paris : Odile Jacob.

Dalla-Bella, S. (2012). "La musique comme outil pour la rééducation". In Bigand, E. et al. . Musique et Cerveau : nouveaux concepts, nouvelles applications. Montpellier : Sauramps Medical. pp. 118-126.

Daumas, C. (2005). "Homme ou femme, le cerveau ne voit pas la différence". Libération. 04/06/05 [ http://www.liberation.fr/week-end/0101531664-hommes-ou-femmes-le-cerveau-ne-voit-pas-ladifference] (vu le 04/09/12).

Ellis, N. C. (1998). "Emergentism, connectionism and language learning". Language Learning, 48 :4. pp. 631-664.

Fonagy, I. (1983). La vive voix : Essais de psycho-phonétique. Paris: Payot.

Fried, R. et Berkowitz, L. (1979). "Music hath charms...and can influence helpfulness". Journal of Applied Social Psychology, 9, pp. 199-208.

Gaffiot, F. (1934). Dictionnaire Latin-Français. Hachette : Paris.

Goody, J. (1994). Entre l'oralité et l'écriture. PUF: Paris.

Graham, C. (2000). Jazz Chants: Old and New. Oxford: Oxford University Press.

Grahn, J. A. (2011). "Advances in neuroimaging techniques: implications for the shared syntactic integration resource hypothesis". Language and Music as Cognitive Systems. Oxford: Oxford University Press.

Guétin, S. et Touchon, J. (2012). "Musicothérapie et prise en charge de la douleur". In Bigand, E. et al.. Musique et Cerveau : nouveaux concepts, nouvelles applications. Montpellier : Sauramps Medical. pp. 127-135.

Guimbretière, É. (1994). Phonétique et enseignement de l'oral. Paris: Didier / Hatier. 
Hesling, I. (2002). "L'hémisphère cérébral droit : un atout en anglais de spécialité". ASp [En ligne]. Consulté le 04 septembre 2012.

Hoch, L. et al. (2008). "Musique, syntaxe et sémantique : des ressources d'intégration structurale et temporelle partagée? ". Revue de Neuropsychologie. vol. 18, n 1-2. pp. 33-59.

Hyman, L. 1975. Phonology: Theory and Practice. New York: Holt.

Jones, D. (1931). "On phonemes". Travaux du Cercle Linguistique de Prague. Nº IV, pp. 74-79.

Krashen, S. D. (1988). Second Language Acquisition and Second Language Learning. London: Prentice Hall.

Iverson, P et Kuhl, P. (1995). "Mapping the perceptual magnet effect for speech using signal theory and multidimensional scaling". Journal of Acoustical Society of America, Volume 94, No 1, January 1995. pp. 553-62.

Kamiyama, T. (2009). "Apprentissage phonétique des voyelles orales du français langue étrangère chez des apprenants". Thèse de Doctorat de phonétique, Option Phonétique Didactique, Université Sorbonne Nouvelle.

Kuhl, P. (2004). "Early language acquisition: cracking the speech code". Nature Reviews Neurosciences, 5. pp. 831-843.

Larsen-Freeman, D. (1997). "Chaos/Complexity Science and Second Language Acquisition". Applied Linguistics, 18 (2), pp. 141-165.

Lauret, B. (2007). Enseigner la prononciation du français : questions et outils. Paris: Hachette.

Liberman, A. \& Cooper, F. \& Shankweiler, D. et Studdert-Kennedy, M. (1967). "Perception of the speech code". Psychological Review, vol. 74. pp. 431-461.

Lisker, L. \& Abramson, A.S. (1964) "A cross-language study of voicing in initial stops: acoustical measurements". Word. 20. pp. 384-422.

Marschark, M., Siple, P., Lillo-Martin, D., Campbell, R., Everhart, V. S. (1997). Relations of language and thought: the view from Sign Language and Deaf Children. NewYork : Oxford University Press.

Martinet, A. (1970). Éléments de linguistique générale. 4ème édition. Paris : Armand Colin.

Mithen, S. (2007). The Singing Neanderthals. Cambridge: Harvard University Press.

Narcy-Combes, J.-P; Bertin, J.-C.; Grave, P. (2010). Second Language Distance Learning and Teaching: Theoretical Perspectives and Didactic Ergonomics. Hershey: Information Science Reference.

O'Grady, W. (2010). "Emergentism" In P. Hogan (dir.), The Cambridge Encyclopedia of the Language Sciences (pp. 274-76). Cambridge: Cambridge University Press.

Patel, A. V. (2007). Music, language and the brain. New York: Oxford University Press.

Peretz, I. et Coltheart, M. (2003). "Modularity of music processing". Nature Neuroscience, Volume 6, Number 8, July 2003. pp. 688-91.

Randall, M. (2007). Memory, Psychology and Second Language Learning. AmsterdamPhiladelphia : John Benjamins Publishing Company.

Renard, R. (2002). Apprentissage d'une langue étrangère-seconde. 2, La phonétique verbo-tonale. Bruxelles : De Boeck Université.

Rizzolatti, G. et Sinigaglia, C. (2006). Les neurones miroirs. Paris : Odile Jacob.

Rolland, Y. (2011). Apprendre à prononcer. Quels paradigmes en didactique des langues? Paris : Belin. 
Saussure (de), F. (1967). Cours de linguistique générale. Originellement publié par Bailly, C. et al. Édition critique par Mauro (de), T. Paris : Payot.

Schiffmann, S. N. (2001). "Le cerveau en constante reconstruction : le concept de plasticité cérébrale". Cahiers de psychologie clinique, 1/2001, N 16. pp. 11-23.

Tripier-Mondancin, O. (2008). "Structuration des programmes d'enseignement musical, en France, de 1925 à 1997". L'Education musicale, (http://www.leducation-musicale.com/), 27.

Troubetzkoy, N.S. (1939). Principes de phonologie. Paris : Klincksieck.

Truchot, C. (1994). Le plurilinguisme européen. Paris: Champion.

Twaddell, W. F.(1935/57). "On Defining the Phoneme". Language Monograph 16, Baltimore. Reprinted in Joos, 1957, pp. 55-80.

Tyne, H. (2012). "Acquisition d'une langue seconde en milieu naturel : contextes, contacts, enjeux". Les migrants face aux langues des pays d'accueil : acquisition en milieu naturel et formation. Lille : Septentrion.

Vaissière, J. (2008). Ivan Fonagy, un pionnier dans l'étude de l'expressivité dans la parole. Conférence donnée dans le cadre du Festival Agora de l'Ircam. 5-20 Juin.

Vygotski, L. (1934). Pensée et langage. $3^{\text {ème }}$ édition parue en 1997. Paris: La Dispute.

Williams, S. \& Hammarberg, B. (1998). Language switches in L3 production: Implications for a polyglot speaking model. Applied Linguistics, № 19. pp. 295-333.

Zatorre, R.J. (2003). "Absolute pitch: a model for understanding the influence of genes and development on neural and cognitive function". Nature Neuroscience, vol. 6, N 7, July. pp. 662-5.

\section{Sitographie}

Larousse en ligne (2009). Consulté en septembre 2012 : http://www.larousse.fr/dictionnaires/ francais/prononciation/64334

Centre National de Ressources Textuelles et Lexicales (2012). Consulté en septembre 2012 : http://www.cnrtl.fr/etymologie/prononciation.

\section{ANNEXES}

\section{Annexe 1 : Questionnaire locuteurs-plurilingues (LPR et LPRN)}

Questionnaire "Apprentissages et langages" : Les langues

Ce questionnaire s'inscrit dans le cadre d'une recherche de doctorat à L'université Paris 3

- Sorbonne Nouvelle.

Cette étude est anonyme

Nous mesurons à travers quelques questions votre relation au language.

La durée totale du questionnaire est de 10-15 mn.

Nous vous recommandons de ne pas revenir sur vos réponses.

\section{Informations générales}


Précisez votre âge :

Vous êtes :

$\square$ Un homme

Une femme

Quelle est votre nationalité :

Dans quel pays vivez-vous actuellement?

Dans quel pays avez-vous majoritairement fait vos études?

Quel est votre plus haut niveau d'étude obtenu?

(un choix possible)

$\square$ Niveau collège

$\square$ Niveau lycée

$\square$ Enseignement supérieur (université, BTS, etc...)

Autre :

Précisez votre situation professionnelle actuelle*

\section{Informations spécifiques}

Quelle langue pratiquez-vous avec votre famille?

Quelle langue pratiquez-vous le plus tous les jours?

Quelles sont les langues étrangères que vous avez apprises?

À quel âge avez-vous appris votre $1^{\text {ère }}$ langue étrangère ?

(un choix possible) 
$0-5$ ans

5-10 ans

$10-15$ ans

15 ans et plus

Pratiquez-vous très régulièrement au moins une de ces langues étrangères dans un contexte naturel ?

parler avec des locuteurs natifs (réel ou virtuel), voyage/séjour dans un des pays, lire des livres, regarder la TV

$\square$ Oui

$\square$ Non

Les langues et vous

Langue maternelle : langue que je parle en famille, que j'ai pratiqué enfant

Langue étrangère : langue apprise tardivement et non pratiquée régulièrement pendant l'enfance dans un contexte social

Exemple de question 2

\begin{tabular}{|l|c|c|c|c|c|}
\hline & $\begin{array}{c}\text { Tout } \\
\text { a fait } \\
\text { d'accord }\end{array}$ & $\begin{array}{c}\text { Plutôt } \\
\text { d'accord }\end{array}$ & $\begin{array}{c}\text { Moyennement } \\
\text { d'accord }\end{array}$ & $\begin{array}{c}\text { Plutôt } \\
\text { pas } \\
\text { d'accord }\end{array}$ & $\begin{array}{c}\text { Pas du } \\
\text { tout } \\
\text { d'accord }\end{array}$ \\
\hline $\begin{array}{l}\text { Parler comme un natif est le } \\
\text { plus important pour bien } \\
\text { parler une langue étrangère }\end{array}$ & $\square$ & $\square$ & $\square$ & $\square$ & $\square$ \\
\hline $\begin{array}{l}\text { Transmettre des émotions } \\
\text { est le plus important pour } \\
\text { bien parler une langue } \\
\text { étrangère }\end{array}$ & $\square$ & $\square$ & $\square$ & $\square$ & $\square$ \\
\hline $\begin{array}{l}\text { Quand je parle dans la langue } \\
\text { maternelle, je pense aux } \\
\text { différentes caractéristiques } \\
\text { de la langue }\end{array}$ & $\square$ & $\square$ & $\square$ & $\square$ & $\square$ \\
\hline $\begin{array}{l}\text { Avant d'utiliser une langue } \\
\text { étrangère à l'oral, je pense } \\
\text { aux différents éléments qui } \\
\text { la composent: } \\
\text { prononciation, intentions, } \\
\text { etc ... }\end{array}$ & $\square$ & $\square$ & $\square$ & $\square$ & $\square$ \\
\hline
\end{tabular}




\begin{tabular}{|c|c|c|c|c|c|}
\hline $\begin{array}{l}\text { Avant de lire un texte dans } \\
\text { une langue étrangère, je } \\
\text { pense aux différents } \\
\text { éléments qui le composent: } \\
\text { prononciation, intention, } \\
\text { etc... }\end{array}$ & $\square$ & $\square$ & $\square$ & $\square$ & $\square$ \\
\hline $\begin{array}{l}\text { Il est plus facile de lire un } \\
\text { texte que de parler } \\
\text { spontanément en langues } \\
\text { étrangères }\end{array}$ & $\square$ & $\square$ & $\square$ & $\square$ & $\square$ \\
\hline $\begin{array}{l}\text { Ma prononciation est } \\
\text { meilleure quand je parle } \\
\text { spontanément à l'oral }\end{array}$ & $\square$ & $\square$ & $\square$ & $\square$ & $\square$ \\
\hline $\begin{array}{l}\text { Ma prononciation est } \\
\text { meilleure quand je lis un } \\
\text { texte }\end{array}$ & $\square$ & $\square$ & $\square$ & $\square$ & $\square$ \\
\hline $\begin{array}{l}\text { J'ai l'impression que ce que } \\
\text { j'entends quand je parle n'est } \\
\text { pas la même chose que ce } \\
\text { qu'entendent mes } \\
\text { interlocuteurs. }\end{array}$ & $\square$ & $\square$ & $\square$ & $\square$ & $\square$ \\
\hline $\begin{array}{l}\text { Lorsque je parle/lis un texte } \\
\text { en langue étrangère, je } \\
\text { ressens des différences } \\
\text { physiques par rapport à } \\
\text { lorsque je parle/lis un texte } \\
\text { en langue maternelle }\end{array}$ & $\square$ & $\square$ & $\square$ & $\square$ & $\square$ \\
\hline $\begin{array}{l}\text { La lecture m'aide à mieux } \\
\text { prononcer }\end{array}$ & $\square$ & $\square$ & $\square$ & $\square$ & $\square$ \\
\hline $\begin{array}{l}\text { La timidité influence la } \\
\text { qualité de l'oral }\end{array}$ & $\square$ & $\square$ & $\square$ & $\square$ & $\square$ \\
\hline $\begin{array}{l}\text { Quand je parle en langue } \\
\text { étrangère, je ne pense pas en } \\
\text { même temps }\end{array}$ & $\square$ & $\square$ & $\square$ & $\square$ & $\square$ \\
\hline
\end{tabular}

\section{Les langues et vous : suite}

\section{Lorsque je change de langue j'ai l'impression :}

(Plusieurs choix possibles)

D'être la même personne 
$\square$ De changer de personnalité

D'agir différemment avec les autres

Lorsque je change de langue j'ai l'impression :

(Un choix possible)

D'être dans le même état d'esprit

$\square$ De changer d'état d'esprit

Je pense que l'écriture et l'oral

(Plusieurs choix possibles)

Sont naturellement liés

Fonctionnent différemment

Fonctionnent de la même manière

Selon moi le important pour bien parler c'est :

(plusieurs choix possible)

$\square$ La qualité de prononciation (voyelles, consonnes, intonation, etc...)

$\square$ Être compris et me sentir à l'aise dans la production

Être à l'aise même s'il y a des erreurs de prononciation

Quand je change de langue :

(Plusieurs choix possible)

$\square$ Je veux garder mon identité personnelle

$\square$ Je veux garder mon identité culturelle

$\square$ Je souhaite intégrer l'ensemble des éléments culturels de la langue étrangère

\section{Annexe 2 : Questionnaire musicien (MSS et MAS)}

Questionnaire "Apprentissages et langages" : La musique

Ce questionnaire s'inscrit dans le cadre d'une recherche de doctorat à L'université Paris 3

- Sorbonne Nouvelle.

Cette étude est anonyme

Nous mesurons à travers quelques questions votre relation au language.

La durée totale du questionnaire est de 10-15 mn.

Nous vous recommandons de ne pas revenir sur vos réponses.

\section{Informations générales}


Précisez votre âge :

Vous êtes :

$\square$ Un homme

Une femme

Quelle est votre nationalité :

Dans quel pays vivez-vous actuellement?

Dans quel pays avez-vous majoritairement fait vos études?

Quel est votre plus haut niveau d'étude obtenu ?

(un choix possible)

$\square$ Niveau collège

$\square$ Niveau lycée

$\square$ Enseignement supérieur (université, BTS, etc...)

Autre :

Précisez votre situation professionnelle actuelle*

\section{Informations spécifiques}

Quels sont les instruments de musique que vous pratiquez régulièrement ?

(Plusieurs choix possible)

$\square$ Les cuivres (trompette, cor, tuba, etc...)

Les bois (flûte, basson, haut bois, etc...)

Les cordes frottées (violon, alto, violoncelle, etc...)

Les percussions (xylophone, triangle, batterie, etc...)

Voix (chant lyrique, rock, métal, folk, etc...)

Les cordes pincées (guitare, harpe, etc...)

Les claviers (piano, clavecin, etc...) 
Autres :

Parmi ces instruments, lequel pratiquez-vous le plus?

Depuis combien de temps pratiquez-vous cet instrument?

Quel type de formation en musique avez-vous majoritairement suivi ?

(un choix possible)

Apprentissage en autonomie

$\square$ Cours particuliers

Conservatoires municipaux, nationaux et équivalents

Conservatoires supérieurs

Autre $: \mu$

A quel âge avez-vous commencé la musique (exemple : jouer d'un instrument, faire du solfège)

(un choix possible)

$\square$ 0-5 ans

5-10 ans

10-15 ans

15 ans et plus

Avez-vous déjà joué dans :

(Plusieurs réponses possibles)

Un orchestre amateur ou professionnel

Une formation (semi) professionnelle (duo, trio, quatuor, etc...)

Un groupe amateur

Autre

\begin{tabular}{|l|c|c|c|c|c|}
\hline \multicolumn{2}{|l|}{ La musique et vous } \\
\hline Selon vous, & $\begin{array}{c}\text { Tout } \\
\text { a fait } \\
\text { d'accord }\end{array}$ & $\begin{array}{c}\text { Plutôt } \\
\text { d'accord }\end{array}$ & $\begin{array}{c}\text { Moyennement } \\
\text { d'accord }\end{array}$ & $\begin{array}{c}\text { Plutôt } \\
\text { pas } \\
\text { d'accord }\end{array}$ & $\begin{array}{c}\text { Pas du } \\
\text { tout } \\
\text { d'accord }\end{array}$ \\
\hline $\begin{array}{l}\text { Un bon musicien doit } \\
\text { seulement reproduire les } \\
\text { intentions du }\end{array}$ & $\square$ & $\square$ & $\square$ & $\square$ & $\square$ \\
\hline
\end{tabular}




\begin{tabular}{|c|c|c|c|c|c|}
\hline $\begin{array}{l}\text { Un bon musicien apporte sa } \\
\text { personnalité dans l'œuvre } \\
\text { qu'il interprète }\end{array}$ & $\square$ & $\square$ & $\square$ & $\square$ & $\square$ \\
\hline $\begin{array}{l}\text { Quand je joue de la musique, } \\
\text { je pense aux différentes } \\
\text { techniques que nécessite ce } \\
\text { type de musique }\end{array}$ & $\square$ & $\square$ & $\square$ & $\square$ & $\square$ \\
\hline $\begin{array}{l}\text { Avant de déchiffrer une } \\
\text { partition/tablure je pense } \\
\text { aux différents éléments de la } \\
\text { partition/tablure, tempo, } \\
\text { rythme, tonalité, etc... }\end{array}$ & $\square$ & $\square$ & $\square$ & $\square$ & $\square$ \\
\hline $\begin{array}{l}\text { Avant de jouer une } \\
\text { partition/tablure, que je } \\
\text { connais, je pense aux } \\
\text { différents éléments de la } \\
\text { partition/tablure, tempo, } \\
\text { rythme, tonalité, etc... }\end{array}$ & $\square$ & $\square$ & $\square$ & $\square$ & $\square$ \\
\hline $\begin{array}{l}\text { Il est plus facile de jouer une } \\
\text { partition/tablure que } \\
\text { d'improviser }\end{array}$ & $\square$ & $\square$ & $\square$ & $\square$ & $\square$ \\
\hline $\begin{array}{l}\text { Ma qualité de jeu est } \\
\text { meilleure lorsque j'improvise }\end{array}$ & $\square$ & $\square$ & $\square$ & $\square$ & $\square$ \\
\hline $\begin{array}{l}\text { Ma qualité de jeu est } \\
\text { meilleure lorsque je joue une } \\
\text { partition }\end{array}$ & $\square$ & $\square$ & $\square$ & $\square$ & $\square$ \\
\hline $\begin{array}{l}\text { J'ai l'impression que ce que } \\
\text { j'entends quand je joue n'est } \\
\text { pas la même chose que ce } \\
\text { qu'entendent les spectateurs }\end{array}$ & $\square$ & $\square$ & $\square$ & $\square$ & $\square$ \\
\hline $\begin{array}{l}\text { Lorsque je joue une } \\
\text { partition/tablure dans un } \\
\text { style, je ressens des } \\
\text { différences physiques par } \\
\text { rapport à lorsque je joue une } \\
\text { partition dans un style } \\
\text { différent }\end{array}$ & $\square$ & $\square$ & $\square$ & $\square$ & $\square$ \\
\hline $\begin{array}{l}\text { La partition/tablure m'aide à } \\
\text { mieux jouer }\end{array}$ & $\square$ & $\square$ & $\square$ & $\square$ & $\square$ \\
\hline $\begin{array}{l}\text { La timidité influence la } \\
\text { qualité du jeu }\end{array}$ & $\square$ & $\square$ & $\square$ & $\square$ & $\square$ \\
\hline
\end{tabular}


Quand je joue je ne pense pas en même temps

La musique et vous : Suite

Lorsque je change de style de musique (classique, baroque, moderne, folk, etc...) j'ai l'impression :

(Plusieurs choix possibles)

D'être la même personne

De changer de personnalité

D'agir différemment avec les spectateurs/les autres musiciens

Lorsque je change de style de musique (classique, baroque, moderne, folk, etc...) j'ai l'impression :

D'être dans le même état d'esprit

De changer d'état d'esprit

Je pense que la partition et l'œuvre jouée :

(Plusieurs choix possibles)

Sont naturellement liées

Fonctionnent différemment

Fonctionnent de la même manière

Selon moi le plus important pour bien jouer c'est :

(Plusieurs choix possibles)

$\square$ La qualité du son (notes, rythmes, etc...)

$\square$ Avoir un retour positif des spectateurs

$\square$ Être à l'aise même s'il y a des erreurs dans mon jeu

Quand je change de genre de musique

(Plusieurs choix possibles)

$\square$ Je veux garder mon identité personnelle

$\square$ Je veux garder mon identité culturelle

$\square$ Je souhaite intégrer l'ensemble des éléments de chaque style 


\section{NOTES}

1. Nous préfèrerons ce terme à enseignement de la prononciation, phonétique corrective, etc.

2. Cf. Théories du chaos (Larsen-Freeman, 1997)

3. Assimilation phonologique :"deux consonnes en contact ne partageant pas le même trait s'assimilent, le trait devenant commun, en fonction de la consonne en position forte" (Lauret, 2007 : 74) ; par exemple : dans "médecin" le /d/ aura tendance à se dévoiser au profit d'un / $\mathrm{t} / \mathrm{au}$ contact de la consonne sourde /s/.

4. Alfred Tomatis développa, dans les années 50, des traitements des troubles de l'audition et du langage basés sur sa théorie de la perception intra-utérine. Sa méthode repose sur une science qu'il appelle "audio-psycho-phonologie» et dont les notions clés ont été, pour la plupart, réfutées tour à tour par des médecins, neurologues, acousticiens, etc. Il fut radié de l'ordre des médecins en 1977.

5. Citation traduite par l'auteur le 05/09/12.

6. L'hypothèse de ressources d'intégration syntaxique partagées.

7. Effet magnétique de la perception : "les stimuli autour d'un prototype phonétique de la langue native sont perceptivement attirés vers le prototype, ce qui réduit la distance perceptive entre le prototype et les autres stimuli" (Kamiyama, 2009)

8. Voice Onset time : intervalle de temps entre le début du relâchement de la consonne et le début du voisement de la voyelle. Il intervient notamment dans la perception voisée ou non-voisée des consonnes occlusives, en anglais par exemple (Lisker and Abramson, 1964).

9. La plupart des individus associent principalement la pensée à de la parole subvocale car plus facilement conscientisée (Marscharkl et al., 1997 : chapitre 1).

10. Nous limiterons cet aspect conclusif au fait que notre étude n'a pas pris en compte les différents niveaux et degrés d'actualité des différentes langues connues et pratiquées (Cf. Williams \& Hammarberg, 1998) au profit d'un sentiment holistique.

\section{RÉSUMÉS}

La médiation de la perception / production des sons en langue étrangère, plus communément appelée "enseignement de la prononciation", est une spécialité particulière dans l'enseignement des langues. Peu de pédagogues sont réellement formés à cette discipline qui, même en L1 (contrairement à la syntaxe ou le lexique), reste majoritairement intuitive.

Notre article propose une vision globale de la performance de prononciation du plan acoustique au plan psychosocial complétée par une étude des liens musique-parole afin de mesurer l'impact de la nature du langage (musical ou verbal) sur les représentations des individus. Cet impact sera étudié à travers une enquête quantitative interdisciplinaire montrant notamment que les musiciens ont une meilleure perception de leurs représentations interne et externe tandis que les locuteurs focalisent sur la validation de l'interaction avec autrui. Cette enquête permettra de proposer des pistes didactiques dans la médiation de la perception / production des sons en langue étrangère. 
Foreign language sound perception and production mediation, widely called "pronunciation teaching", has a particular status in language teaching. Very few teachers are really trained for this discipline, which is, even in L1 (and contrary to syntax or lexicon), mainly intuitive.

Our paper aims to reflect a global view of the performance of pronunciation involving both acoustics and psychosocial factors. The paper also studies the links between music and speech in order to measure the impact of the kind of language (music or discourse) on individual representations. This impact will be studied through a quantitative and interdisciplinary survey showing that musicians have a better perception of their internal and external representations whereas speakers are mainly involved in interacting with others. This survey will allow us to propose didactic possibilities for foreign language sound perception and production mediation.

\section{INDEX}

Mots-clés : musique, parole, FLE, prononciation

Keywords : music, speech, pronunciation, French as a Foreign Language

\section{AUTEUR}

\section{GRÉGORY MIRAS}

Grégory Miras est doctorant au laboratoire de Didactique des Langues, des Textes et des Cultures (Diltec) et au Laboratoire de Phonétique et Phonologie (LPP) à l'Université Sorbonne Nouvelle Paris 3. Il travaille sur les liens perceptifs entre la musique instrumentale et la parole afin de proposer des tâches favorisant un travail sur le son plutôt que le sens dans la médiation de la perception et production des sons en langue étrangère.

Courriel : gy.miras@gmail.com.

Toile : https://sites.google.com/site/gregorymiras/.

Adresse : Université Sorbonne Nouvelle - Paris 3, 46, rue Saint-Jacques, 75005 Paris. 